\title{
The Sufficient Condition for the Original Function of the No Zero Entire \\ Function is Infinite
}

\author{
Hesong Yang ${ }^{1, a}$ \\ ${ }^{1}$ Dehong Teachers' College, Yunnan, Dehong 678400 \\ ${ }^{a}$ email
}

Key words: Entire function, Magnitude, Zero

\begin{abstract}
This paper proves that under certain conditions, so that there is an infinite number of zeros for $F(z)=\int_{0}^{z} \sum_{j=1}^{m} e^{\alpha_{j}(w)} d w$.
\end{abstract}

\section{Introduction}

The zero of the entire function is an important research topic in the theory of complex functions. We know that the entire function has infinitely many zeros and easy to get when the is very polynomial time. There must be an infinite number of zeros in the $F(z)=\int_{0}^{z} e^{\alpha(w)} d w$. And in general, for the special polynomial $\alpha(z)$, the zero point of is very much. For example, for $Q(z)=\int_{0}^{z} e^{-w^{n}} d w(n \geq 2)$, there are (see literature). In the research of value distribution, the problem of the existence of the zero of the entire function, such as (which is as the entire function), is often confronted with the problem of the existence of the density of the zeros.

\section{.Problem Presentation and main result}

In 2004, C.C.Yang presented the following questions in the literature [4]:

If is a non-constant entire fuction, is a point in the area. So does have to have infinitely many zeros?

Background of the problem:

If is the entire function, then the entire function does not have zero and you can find the entire function $\alpha(z)$, so that does not have an zero function. So when the is a non-constant entire fuction, the number of zeros will be how to do?

We can easily get the above questions:

(1) $\alpha(z)$ is a non-constant polynomials, is a point in the area, then has infinitely many zeros;

( 2 ) $\alpha(z)$ is a transcendental entire function, and $\rho(\alpha) \in$ 的 $/, F(z)=\int_{z_{0}}^{z} e^{\alpha(w)} d w$ also has infinitely many zeros.

We give a negative answer to the question above. 
We have proved that under certain conditions $F(z)=\int_{0}^{z} e^{a(w)} d w$, has a finite zero. So in what circumstances there are infinite number of zeros it? On this issue, we have the following results:

Lemmas: Set is not less than 2 of the positive integer, is a very integer function of $m$, ream $T^{*}(r)=\max _{1 \leq j \leq m} T\left(r, e^{\alpha_{j}}\right)(\forall r \in I)$, if $\exists j, k \in\{1,2$, 廌, $m\}$ and $I \subset$ 国, make mesI $=+\infty$, and $T\left(r, e^{\alpha_{j}-\alpha_{k}}\right)>\frac{1}{5} T^{*}(r)(\forall r \in I)$, then has infinitely many zeros.

\section{Related lemmas}

Lemmas $1^{[1]}$ Set for the very number of the entire function, $f(z)=e^{\beta(z)}$, so

$$
T(r, \beta)+T\left(r, \beta^{\prime}\right)=o(T(r, f))(r \rightarrow \infty) .
$$

Lemmas $2^{[3]}$ set integer, is a linearly independent integral function of $m$, for $\forall z \in[\underline{W}$, it has $\max _{1 \leq j \leq m}\left|g_{j}(z)\right|>0$, ream $T(r)=\frac{1}{2 \pi} \int_{0}^{2 \pi} u\left(r e^{i \theta}\right)-u(0), \quad$ one of it $u(z)=\sup _{1 s j s m} \log \left|g_{j}(z)\right|$, when $g_{m+1}(z)=g_{1}(z)+g_{2}(z)+凶+g_{m}(z)$, it has $T(r) \leq \sum_{j=1}^{m+1} N_{m-1)}\left(r, \frac{1}{g_{j}}\right)+S(r)$, where meets enables to be beyond the sub pure function, so $S(r)=o(T(r))(r \rightarrow+\infty ; r \notin E$, mes $E<+\infty)$

Lemmas $3^{[3]}$ Under the condition of the lemma 2 , for $\forall j, k \in\{1,2$, 网, $m\}$, it has

$$
\begin{gathered}
T\left(r, g_{j} / g_{k}\right) \leq T(r)+O(1)(r \rightarrow \infty), \\
N\left(r, 1 / g_{j}\right) \leq T(r)+O(1)(r \rightarrow \infty) .
\end{gathered}
$$

Lemmas 4 If there is a transcendental entire function $\partial(z)$, the polynomial $\mathrm{P}(z)$ and the entire function $\beta(z)$, fulfilled $F(z)=\int_{0}^{z} e^{\alpha(w)} d w=\mathrm{P}(z) e^{\beta(z)}$, so $\rho(\alpha(z))=\rho(\beta(z))$.

Demondstrate of on both sides of the derivative.

Thus $T\left(r, e^{\alpha(z)}\right) \leq T\left(r, e^{\beta(z)}\right)+o\left(T\left(r, e^{\beta(z)}\right)\right)$

Therefore $T\left(r, e^{\alpha(z)}\right) \leq T\left(r, e^{\beta(z)}\right)(1+o(1))$

From lemma $3 \rho(\alpha) \leq \rho(\beta)$

From formula $e^{\beta(z)}=\frac{e^{\alpha(z)}}{P^{\prime}(z)+P(z) \beta^{\prime}(z)}$

In the same way $T\left(r, e^{\beta(z)}\right) \leq T\left(r, e^{\alpha(z)}\right)+o\left(T\left(r, e^{\beta(z)}\right)\right)$

So $(1-o(1)) T\left(r, e^{\beta(z)}\right) \leq T\left(r, e^{\alpha(z)}\right)$

From lemmas $3 \rho(\beta) \leq \rho(\alpha)$

So $\rho(\alpha)=\rho(\beta)$ 


\section{Proof of Theorem}

Assuming that is at most only finite zeros, note that $F(0)=0$, So there must be an entire function and polynomial to make $\operatorname{deg}(P) \geq 1$, and $\int_{0}^{z} \sum_{j=1}^{m+1} e^{\alpha_{j}(w)} d w=P(z) e^{\beta(z)},(1)$

On the sides of derivation $\sum_{j=1}^{m} e^{\alpha_{j}(z)}=\left\{P^{\prime}+P \beta^{\prime}\right\} e^{\beta(z)}$, (2)

Thus there $\sum_{j=1}^{m} e^{\alpha_{j}(z)-\beta(z)}-P^{\prime}(z)=P(z) \beta^{\prime}(z),(3)$

Without loss of generality, can be set $\beta^{\prime}(z) \neq 0$, is linearly independent, And used to replace. There exist $r_{0}>0$, so when $|z|>r_{0},\left|P^{\prime}(z)\right| \geq 1$, make $T(z)=\frac{1}{2 \pi} \int_{0}^{z} u\left(r e^{\circledast \theta}\right) d \theta-u(0)(r \in I)$.

There is $u(z)=\log \max _{1 \leq j \leq m+1}\left|g_{j}(z)\right|$

Because of is a linear combination of admissible $m+2$, Therefore, by the Cartan lemma

$$
\begin{aligned}
& T(r)<N_{m]}\left(r, \frac{1}{P^{\prime}}\right)+N_{m]}\left(r, \frac{1}{P \beta^{\prime}}\right)+o\{T(r)\}(r \rightarrow \infty, r \notin E) . \\
& \text { So is }(1-o(1)) T(r) \leq O(\log r)+o\left(T\left(r, e^{\beta}\right)\right),(4)
\end{aligned}
$$

From formula (2)

$$
\begin{aligned}
& T\left(r, e^{\beta}\right) \leq T\left(r, \frac{1}{P^{\prime}+P \beta^{\prime}}\right)+T\left(r, \sum_{j=1}^{m+1} e^{\alpha_{j}}\right) \\
& \leq T\left(r, \frac{1}{P^{\prime}+P \beta^{\prime}}\right)+\sum_{j=1}^{m+1} T\left(r, e^{\alpha_{j}}\right)+\log (m+1)
\end{aligned}
$$

Thus the binding lemma

$$
(1-o(1)) T\left(r, e^{\beta}\right) \leq \sum_{j=1}^{m+1} T\left(r, e^{\alpha_{\jmath}}\right) \leq(m+1) T^{*}(r),
$$

From and lemmas 3

$$
\frac{1}{5} T^{*}(r)<T\left(r, e^{\alpha_{j}-\alpha_{k}}\right) \leq T(r)+O(1)(r \rightarrow \infty) .(6)
$$

From formula (4) (6)

$$
\frac{1-o(1)}{5} T^{*}(r) \leq O(\log r)+o\left\{T\left(r, e^{\beta}\right)\right\},
$$

So it has

$T^{*}(r)=O(\log r)$ contradiction,

So must have infinitely many zeros.

\section{Conclusion}

In the above theorem, we obtain a sufficient condition for the integral function of the form, such as $F(z)=\int_{0}^{z} \sum_{j=1}^{m} e^{\alpha_{j}(w)} d w$, to have infinitely many zeros. Under what conditions, we can obtain a neces- 
sary and sufficient condition for the to have infinite zeros, and we will make further research on this problem.

\section{Reference}

[1] Le Yang,values distribution theory and its new research,Science Press [M],1982.

[2] Honxun Yi, Chongjun Yang,Uniqueness of Meromorphic Function,Science Press [M],1995.

[3] Henri Cartan. The strength of cartan's version of nevanlinna theory[J]. London Math.Soc.2004,36:433-454.

[4] Yang Chungchun. On the value distribution of derivatives[J]. Indian Math, 2004, 35(8):10271031.

[5] A.Escassut, W. Tutschke C.C.Yang. Some Topics on value Distribution and Differentiability in Complex and P-adic Analysis[M].Mathematics Monograph Series 11.Beijing:science press.2008,400-401.

[6] Shengguang Bai, entire functions and Meromorphic Function, Huazhong Normal University press [M],1989.

[7] J.Miles, On entire functions of infinite order with radially distributed zeros, Pacific.J.Math,81(1979),131-157.MR.801:30046.

[8] W.K.Hayman, Meromorphic Function[M], Carendon Press, Oxford, 1964.

[9] L.A.Rubel and B.A.Taylor, A Fourier series method for meromorphic and entire functions , Bull.Soc.Math.France[J],V.96,1968,53-96. 